\title{
Icariin regulates systemic iron metabolism by increasing hepatic hepcidin expression through Stat3 and Smad1/5/8 signaling
}

\author{
MIAO ZHANG ${ }^{1,2^{*}}$, JING LIU $^{2 *}$, WENLI GUO ${ }^{2}$, XIN LIU ${ }^{1}$, SIJIN LIU $^{2,3}$ and HUIJUN YIN ${ }^{1,4}$ \\ ${ }^{1}$ Department of Cardiovascular Diseases, Beijing Xiyuan Hospital, China Academy of Chinese Medical Sciences, Beijing 100091; \\ ${ }^{2}$ State Key Laboratory of Environmental Chemistry and Ecotoxicology, Research Center for Eco-Environmental Sciences, \\ Chinese Academy of Sciences, Beijing 100085; ${ }^{3}$ Institute of Environment and Health, Jianghan University, Wuhan, \\ Hubei 430056; ${ }^{4}$ Gansu University of Traditional Chinese Medicine, Lanzhou, Gansu 730000, P.R. China
}

Received December 11, 2015; Accepted March 3, 2016

DOI: $10.3892 /$ ijmm.2016.2545

\begin{abstract}
Systemic iron homeostasis is strictly controlled under normal conditions to ensure a balance between the absorption, utilization, storage and recycling of iron. The hepcidin-ferroportin (FPN) axis is of critical importance in the maintenance of iron homeostasis. Hepcidin deficiency gives rise to enhanced dietary iron absorption, as well as to increased iron release from macrophages, and this in turn results in iron accumulation in the plasma and organs, and is associated with a range of tissue pathologies. Low hepcidin levels have been demonstrated in most forms of hereditary hemochromatosis ( $\mathrm{HH})$, as well as in $\beta$-thalassemia. Therapies that increase hepcidin concentrations may potentially play a role in the treatment of these iron overload-related diseases. To date, natural compounds have not been extensively investigated for this purpose, to the best of our knowledge. Thus, in the present study, we screened natural compounds that have the potential to regulate hepcidin expression. By performing hepcidin promoter-luciferase assay, RT-qPCR and animal experiments, we demonstrated that icariin and berberine were potent stimulators of hepcidin transcription.
\end{abstract}

Correspondence to: Professor Sijin Liu, State Key Laboratory of Environmental Chemistry and Ecotoxicology, Research Center for Eco-Environmental Sciences, Chinese Academy of Sciences, 18 Shuangqing Road, Beijing 100085, P.R. China

E-mail: sjliu@rcees.ac.cn

Professor Huijun Yin, Department of Cardiovascular Diseases, Beijing Xiyuan Hospital, China Academy of Chinese Medical Sciences, Beijing 100091, P.R. China

E-mail: huijunyin@aliyun.com

"Contributed equally

Abbreviations: $\mathrm{HH}$, hereditary hemochromatosis; Hamp1 ${ }^{-/}$, hepcidin-deficient; FPN, ferroportin; HFE, human hemochromatosis protein; TFR2, transferrin receptor 2; DMSO, dimethyl sulfoxide; FBS, fetal bovine serum; GAPDH, glyceraldehyde 3-phosphate dehydrogenase; Wt, wild-type; LPS, lipopolysaccharide; H\&E, hematoxylin and eosin

Key words: hepcidin, iron, metabolism, natural compounds, icariin
Mechanistic experiments indicated that icariin and berberine increased hepcidin expression by activating the signal transducer and activator of transcription 3 (Stat3) and Smad1/5/8 signaling pathways. The induction of hepcidin was confirmed in mice following icariin administration, coupled with associated changes in serum and tissue iron concentrations. In support of these findings, the icariin analogues, epimedin A, B and C, also increased hepatic hepcidin expression. However, these changes were not observed in hepcidin-deficient $\left[\mathrm{Hampl}^{-/-}\right.$or Hamp1-knockout (KO)] mice following icariin administration, thereby verifying hepatic hepcidin as the target of icariin. Although berberine exhibited a robust capacity to promote hepcidin expression in vitro, it failed to alter hepcidin expression in mice. Taken together, the findings of the present study suggest that icariin exhibits a robust capacity to increase hepatic hepcidin expression and to modulate systemic iron homeostasis. The present study therefore highlights the significance of using natural compounds to ameliorate iron disorders through the regulation of hepcidin expression.

\section{Introduction}

Iron is an essential metal involved in a large array of biological processes, such as energy metabolism, DNA replication and other important cellular functions (1-3). Systemic iron homeostasis is accurately and strictly governed to ensure a balance between iron absorption, utilization and storage in mammals (4). Hepcidin is the central hormone secreted by hepatocytes that plays a fundamental role in the regulation of iron flow through limiting dietary iron absorption from the duodenum and reducing iron egress from macrophages (5). Hepcidin achieves this effect by binding to its receptor, ferroportin (FPN; the only known iron exporter thus far), on the plasma membrane of target cells and inducing its internalization and degradation (5). The misregulation of hepcidin gives rise to several types of iron disorders, including refractory iron deficiency anemia, as well as iron overload-related diseases, such as hereditary hemochromatosis $(\mathrm{HH})$ and $\beta$-thalassemia (6). $\mathrm{HH}$ is characterized by enhanced iron absorption and, consequently, iron overload in various organs due to the low hepcidin levels (7). Hepcidin deficiency derives from genetic mutations in hepcidin itself, or in other genes encoding its upstream regulators, such 
as the human hemochromatosis protein (HFE) and transferrin receptor 2 (TFR2) (6). Insufficient hepcidin levels result in elevated FPN concentrations and this facilitates dietary iron absorption and increases iron egress from macrophages (8). As a result, excess iron accumulates in the liver and other organs, eventually leading to iron overload and tissue injuries. $\beta$-thalassemia is also a type of iron overload-related disease characterized by low hepcidin levels, although the mechanisms involved differ somewhat from those leading to HH (9-11). Therefore, strategies that are able to increase the circulating hepcidin concentrations may be promising therapies for iron overload-related diseases, including $\mathrm{HH}$ and $\beta$-thalassemia. For this purpose, hepcidin mimics and agonists are under development (8).

Exhaustive and ongoing efforts are being made in search of natural botanical compounds for use in the treatment of a wide spectrum of disorders, including various types of cancer and hematological diseases. Sometimes drugs derived from natural compounds often exhibit greater efficacy than drugs manufactured synthetically. To date, to the best of our knowledge, no extract or natural botanical compound has been found that can upregulate hepcidin expression. To this end, in the present study, we assessed 12 pure natural compounds with the potential to modulate iron metabolism. All these compounds are extracts from traditional Chinese herbal medicinal plants, and many of them have been reported to be effective therapeutically for multiple disorders, including inflammation, oxidative injuries and cardiovascular diseases (12-20). According to the Chinese Pharmacopoeia (21), all of these compounds are capable of improving microcirculation and have the potential to modulate blood cell formation. Thus, we deliberately selected these compounds in the present study. Of these compounds, icariin was found to markedly stimulate hepcidin expression in vitro and in vivo. Mechanistic experiments indicated that icariin increased hepcidin transcription through a combination of the activation of the signal transducer and activator of transcription 3 (Stat3) and Smad1/5/8 signaling pathways. Our combined data therefore suggest that icariin represents a promising therapeutic strategy for restricting iron absorption and iron egress from macrophages, and hence for ameliorating iron overloadrelated diseases by regulating hepcidin-FPN signaling.

\section{Materials and methods}

Chemicals and reagents. The following pure natural compounds extracted from Chinese medicinal plants were purchased from the National Institutes for Food and Drug Control of China (Beijing, China) with $>99.5 \%$ purity: propyl gallate, $\mathrm{C}_{10} \mathrm{H}_{12} \mathrm{O}_{5}$; resveratrol, $\mathrm{C}_{14} \mathrm{H}_{12} \mathrm{O}_{3}$; astragaloside, $\mathrm{C}_{41} \mathrm{H}_{68} \mathrm{O}_{14}$; curcumin, $\mathrm{C}_{21} \mathrm{H}_{20} \mathrm{O}_{6}$; paeoniflorin, $\mathrm{C}_{23} \mathrm{H}_{28} \mathrm{O}_{11}$; ligustrazine, $\mathrm{C}_{8} \mathrm{H}_{12} \mathrm{~N}_{2} \cdot \mathrm{HCl} \cdot 2 \mathrm{H}_{2} \mathrm{O}$; ferulic acid, $\mathrm{C}_{10} \mathrm{H}_{10} \mathrm{O}_{4}$; ginsenoside $\mathrm{Rb} 1, \mathrm{C}_{54} \mathrm{H}_{92} \mathrm{O}_{23}$; wogonin, $\mathrm{C}_{16} \mathrm{H}_{12} \mathrm{O}_{5}$; liquiritin, $\mathrm{C}_{21} \mathrm{H}_{22} \mathrm{O}_{9}$; berberine, $\mathrm{C}_{20} \mathrm{H}_{18} \mathrm{NO}_{4}$; icariin, $\mathrm{C}_{33} \mathrm{H}_{40} \mathrm{O}_{15}$; epimedin $\mathrm{A}, \mathrm{C}_{39} \mathrm{H}_{50} \mathrm{O}_{19}$; epimedin $\mathrm{B}, \mathrm{C}_{38} \mathrm{H}_{48} \mathrm{O}_{19}$; and epimedin $\mathrm{C}, \mathrm{C}_{39} \mathrm{H}_{50} \mathrm{O}_{19}$. Dimethyl sulfoxide (DMSO; Solarbio, Beijing, China) was used to dissolve these compounds, and the final concentration of DMSO in the culture medium was $<0.5 \%$.

Cell culture and treatments. The human hepatocellular carcinoma cell line, HepG2, and the mouse hepatocellular carcinoma cell line, Hepa 1-6, obtained from the Institute of Biochemistry and Cell Biology, Shanghai Institute for Biological Sciences, Chinese Academy of Sciences were cultured in RPMI-1640 and Dulbecco's modified Eagle's medium (DMEM), respectively, supplemented with $10 \%$ heat-inactivated fetal bovine serum (FBS) (all from Gibco, Grand Island, NY, USA) at $37^{\circ} \mathrm{C}$ under a humidified atmosphere with $5 \% \mathrm{CO}_{2}$, as previously described $(22,23)$. The cells were collected for RNA or protein extraction $24 \mathrm{~h}$ after being subjected to the various treatments (the cells were treated with the various compounds at concentrations of $5 \mu \mathrm{M}$ for $24 \mathrm{~h}$ ). Untreated (control) cells were cultured with the corresponding DMSO-containing medium.

Cytotoxicity assay. The cytotoxicity of the natural compounds was evaluated using an MTT assay kit according to the manufacturer's instructions (Roche Life Science, Basel, Switzerland). Briefly, the HepG2 and the Hepa 1-6 cells were first serum-starved overnight, then inoculated into 96-well plates with 5,000 cells/well. The cells were then subjected to the different treatments. The cells were cultured for an additional $24 \mathrm{~h}$, and subsequently, $20 \mu \mathrm{l}$ MTT solution $(5 \mathrm{mg} / \mathrm{ml})$ was added to each well, followed by incubation for a further $4 \mathrm{~h}$. At the end of the incubation period, $200 \mu \mathrm{l}$ DMSO were added to each well, and the absorbance at $490 \mathrm{~nm}$ was measured using a microplate reader (Thermo Fisher Scientific, Waltham, MA, USA).

Luciferase assay. The HepG2 and the Hep 1-6 cells were seeded at $2 \times 10^{5}$ cells/well in a 24 -well plate $12 \mathrm{~h}$ prior to transfection. The cells were then co-transfected with $0.8 \mu \mathrm{g}$ of a hepcidin-promoter-luciferase plasmid and $80 \mathrm{ng}$ of Renilla luciferase plasmid with Lipofectamine 2000 in accordance with the manufacturer's instructions (Invitrogen, Carlsbad, CA, USA). The medium was replaced with RPMI-1640 or DMEM containing 10\% FBS $6 \mathrm{~h}$ later, and the cells were then treated with each compound (at $5 \mu \mathrm{M}$ ) for $24 \mathrm{~h}$. Afterwards, the cells were collected and washed with PBS, and finally lysed in lysis buffer (Promega, Madison, WI, USA). The cell lysates were then assessed for luciferase activity using the Dual-Luciferase Reporter assay system (Promega). The relative firefly luciferase activity for each sample was normalized to that of Renilla luciferase.

$R N A$ extraction and $R T-q P C R$. Total RNA was purified from the cells using TRIzol reagent according to the manufacturer's instructions (Invitrogen Grand, NY, Island, USA). Total RNA from liver specimens (obtained from mice, as described below) was also extracted using TRIzol reagent according to the manufacturer's instructions after the specimens were pulverized in liquid nitrogen. The mRNA expression levels were assessed by performing qPCR using SYBR-Green qPCR Master Mix (Qiagen, Valencia, CA, USA). Glyceraldehyde 3phosphate dehydrogenase (GAPDH) was used as a housekeeping gene for the normalization of relative gene expression. The primer sequences used were as follows: human hepcidin forward, 5'-CCTGACCAGTGGCTCTGTTT-3' and reverse, 5'-CACATCCCACACTTTGATCG-3'; human GAPDH forward, 5'-GAAGGTGAAGGTCGGAGT-3' and reverse, 5'-GAAGATGGTGATGGGATTTC-3'; mouse hepcidin forward, 5'-CTGAGCAGCACCACCTATCTC-3' and reverse, 
5'-TGGCTCTAGGCTATGTTTTGC-3'; and mouse GAPDH forward, 5'-AAGGTCATCCCAGAGCTG' and reverse, 5'-GCC ATGAGGTCCACCACCCT-3'.

Western blot analysis. Following treatment with the compounds, HepG2 cells were harvested in cold PBS, and total proteins were then extracted using RIPA lysis buffer (Solarbio) supplemented with protease inhibitor cocktail (Roche Life Science). Interleukin (IL)- 6 was used as positive control for p-Stat 3 detection, which was purchased from Sigma Aldrich. Proteins were extracted following $24 \mathrm{~h}$ of administration at $100 \mathrm{ng} / \mathrm{ml}$. Equal amounts of protein lysates for each sample were subjected to SDS-PAGE and western blot analysis following a standard procedure, as previously described $(24,25)$. The primary antibodies used were as follows: anti-p-Smad1/5/8 (1:1,000; sc-12353) and anti-Smad1 (1:1,000; sc-7965) antibodies (both from Santa Cruz Biotechnology, Santa Cruz, CA, USA); antip-Stat3 $(1: 1,000 ; 9145)$ and anti-Stat3 $(1: 1,000 ; 9139)$ antibodies (both from Cell Signaling Technology, Danvers, MA, USA); and anti-GAPDH (1:2,000; G8795; Sigma-Aldrich, St. Louis, MO, USA). Protein quantification was performed using Image J software (NIH, http://rsb.info.nih.gov/ij/).

Animal experiments. All animal care and experimental procedures were approved by the Animal Ethics Committee at Xiyuan Hospital, China Academy of Chinese Medical Sciences (Beijing, China). Eight-week-old mice were used in this study. Wild-type (Wt) ICR mice were purchased from the Laboratory Animal Technology Co., Ltd. (Beijing, China), and housed in a central specific pathogen-free (SPF) facility at the Beijing Xiyuan Hospital. Hepcidin-deficient $\left[\mathrm{Hamp1}^{-1-}\right.$ or Hamp1-knockout (KO)] mice were originally provided by Dr Sophie Vaulont [Institut National de la Santé et de la Recherche Médicale (INSERM)] (26) and have been backcrossed into the C57BL/6 background (27). To deplete iron, the $\mathrm{Hamp1}^{-/-}$mice were placed on a low-iron diet $(4 \mathrm{ppm}$ iron) 1 week prior to the administration of the compounds. This method was developed to reduce the serum iron content in the Hamp1 $1^{-/}$mice in order to more clearly demonstrate changes in serum iron levels following the administration of the compounds. The mice were randomly grouped with 8 mice in each group $(n=8)$. The compounds were administered intraperitoneally to the mice at a dose of $100 \mathrm{mg}$ / $\mathrm{kg}$ body weight. The control mice received PBS containing DMSO at the relevant concentration. For the short-term treatment experiments, the mice were sacrificed 6 or $48 \mathrm{~h}$ after the injection of the compounds. With respect to the long-term treatments, the compounds were administered to the mice every 2 days, and the mice were sacrificed on days 4,8 or 16 . Lipopolysaccharide (LPS) was used as a positive control and was purchased from Sigma-Aldrich. Mice were administrated with LPS at $100 \mu \mathrm{g} /$ mouse for $6 \mathrm{~h}$. Blood was collected, and liver and spleen specimens from each mouse were isolated and individually separated. A small section of each liver specimen was quickly frozen in liquid nitrogen and then stored at $-80^{\circ} \mathrm{C}$ for future RNA extraction.

Iron parameters. The serum iron levels were determined using a serum iron detection kit according to the manufacturer's instructions (Nanjing Jiancheng Bioengineering Institute,
Nanjing, China). Hepatic and spleen iron contents were determined following the established protocols in our laboratory, as previously described (25). Briefly, liver and spleen specimens were first digested with an acid solution at $65^{\circ} \mathrm{C}$ for $72 \mathrm{~h}$. Thereafter, chromagen working solution was added to each supernatant. Finally, the absorption at $535 \mathrm{~nm}$ was measured using a microplate reader (Thermo Fisher Scientific).

Histological examination. The liver specimens were fixed in $10 \%$ buffered formaldehyde, and embedded in paraffin. The deparaffinized sections were stained with hematoxylin and eosin (H\&E), following a standard protocol, as previously described $(25,28,29)$.

Statistical analysis. The SPSS 17.0 statistics package was used to analyze the experimental data. One-way analysis of variance (ANOVA) was used to differentiate the mean differences among groups compared to the control group. The differences between 2 groups were determined using an independent Student's t-test. All experimental data are presented as the means \pm SD. A P-value $<0.05$ was considered to indicate a statistically significant difference.

\section{Results}

Screening of natural compounds for modulating hepcidin expression. The liver (where hepatocytes secrete hepcidin) is the predominant organ for the secretion of hepcidin into the circulation in order to regulate iron homeostasis (30). Thus, in this study, we used liver-derived cell lines. HepG2 and Hepa 1-6 are established hepatocyte cell lines representative of human cells and mouse cells, respectively. In an effort to investigate the potential role of natural compounds in the modulation of hepcidin expression, we screened 12 pure natural products using the dual hepcidin luciferase assay. The reporter construct was developed by our research group with the human hepcidin promoter fragment $(1.6 \mathrm{~kb})$ upstream of the firefly luciferase gene, as previousy described (31). The screening assay was performed in both the HepG2 and Hepa 1-6 cells. Prior to screening, cytotoxicity was evaluated by MTT assay. All these compounds exhibited no significant toxicity to both the HepG2 and Hepa 1-6 cells, with only a mild reduction in cell viability observed at higher concentrations of berberine, curcumin, icariin and wogonin (data not shown). Thus, we used a concentration of $5 \mu \mathrm{M}$ in the initial screening assay, and cell viability was not impaired by any of the compounds (data not shown). Following treatment with the compounds for $24 \mathrm{~h}$, hepcidin-luciferase activity was measured. As shown in Table I, propyl gallate, resveratrol, berberine, icariin and wogonin were observed to enhance hepcidin-luciferase activity by $>2$-fold in the HepG2 cells, compared to the untreated controls $(\mathrm{P}<0.05)$. Astragaloside, ligustrazine, ginsenoside $\mathrm{Rb} 1$ and liquiritin were found to enhance hepcidin-luciferase activity by approximately $50 \%$ in the HepG2 cells compared with the untreated controls $(\mathrm{P}<0.05)$. However, curcumin, paeoniflorin and ferulic acid failed to significantly alter hepcidin-luciferase activity in the HepG2 cells $(\mathrm{P}>0.05)$. Analogously, in the Hepa 1-6 cells, berberine, icariin and wogonin promoted hepcidin-luciferase activity by $>2$-fold compared with the untreated controls $(\mathrm{P}<0.05)$, 
Table I. Changes in relative luciferase activity in cells treated with the various compounds compared to the untreated controls.

\begin{tabular}{lcc}
\hline Compounds & HepG2 & Hepa 1-6 \\
\hline Propyl gallate & $\uparrow \uparrow \uparrow \uparrow^{\mathrm{a}}$ & - \\
Resveratrol & $\uparrow \uparrow \uparrow \uparrow^{\mathrm{a}}$ & - \\
Astragaloside & $\uparrow^{\mathrm{a}}$ & - \\
Curcumin & - & - \\
Paeoniflorin & - & - \\
Ligustrazine & $\uparrow^{\mathrm{a}}$ & - \\
Ferulic acid & - & $\uparrow^{\mathrm{a}}$ \\
Ginsenoside Rb1 & $\uparrow^{\mathrm{a}}$ & $\uparrow^{\mathrm{a}}$ \\
Liquiritin & $\uparrow^{\mathrm{a}}$ & $\uparrow^{\mathrm{a}}$ \\
Berberine & $\uparrow \uparrow \uparrow \uparrow^{\mathrm{a}}$ & $\uparrow \uparrow \uparrow \uparrow^{\mathrm{a}}$ \\
Icariin & $\uparrow \uparrow \uparrow \uparrow^{\mathrm{a}}$ & $\uparrow \uparrow \uparrow \uparrow^{\mathrm{a}}$ \\
Wogonin & $\uparrow \uparrow \uparrow \uparrow^{\mathrm{a}}$ & $\uparrow \uparrow \uparrow \uparrow^{\mathrm{a}}$ \\
\hline
\end{tabular}

Arrows represent changes in relative luciferase activity as follows: 1 arrow, $\sim 50 \%$ increase; and 4 arrows, $>200 \%$ increase. Dash (-) indicates no significant change. Three independent experiments were performed with 6 biological replicates $(n=6) .{ }^{a} \mathrm{p}<0.05$, compared to the untreated control.

which was consistent with the results observed in the HepG2 cells. Ferulic acid, ginsenoside Rb1 and liquiritin were able to stimulate hepcidin-luciferase activity by approximately $50 \%$ in the Hepa 1-6 cells in comparison with the untreated cells $(\mathrm{P}<0.05)$. The remaining compounds (propyl gallate, resveratrol, astragaloside, curcumin, paeoniflorin and ligustrazine) failed to significantly alter the hepcidin-luciferase activity in the Hepa 1-6 cells $(\mathrm{P}>0.05)$. Taken together, these findings indicated that berberine, icariin and wogonin exerted a robust stimulatory effect on hepcidin transcription in both the HepG2 and Hepa 1-6 cells. Thus, these 3 compounds were selected for use in our subsequent experiments.

Validation of screening data using RT-qPCR. To confirm the screening results described above, RT-qPCR was employed to determine the hepcidin mRNA levels following treatment with the selected compounds. As shown in Fig. 1, hepcidin expression in the HepG2 cells was increased by approximately 2 -fold by icariin at a concentration of $5 \mu \mathrm{M}$, and was further increased at concentrations of 20 and $50 \mu \mathrm{M}$ by approximately 3-fold, compared with the untreated cells $(\mathrm{P}<0.05)$. Similarly, hepcidin expression in the Hepa 1-6 cells was induced by icariin (at concentrations of 5,20 and $50 \mu \mathrm{M}$ ) in a dose-dependent manner, with a maximum increase observed at the concentration of $50 \mu \mathrm{M}$ of approximately 3 -fold compared to the controls (Fig. 1; $\mathrm{P}<0.05$ ). Analogously, berberine increased hepcidin expression in the HepG2 cells by $>3$-fold at both 5 and $50 \mu \mathrm{M}$ compared with the untreated cells (Fig. $1 ; \mathrm{P}<0.05$ ). In parallel to the HepG2 cells, hepcidin expression was elevated by approximately 4 -fold in the Hepa 1-6 cells by berberine at both 5 and $50 \mu \mathrm{M}$ (Fig. 1; $\mathrm{P}<0.05$ ). By contrast, wogonin failed to alter hepcidin expression, and this was revealed by RT-qPCR (data not shown). Based on these results, the stimulatory effects of icariin and berberine on hepcidin expression

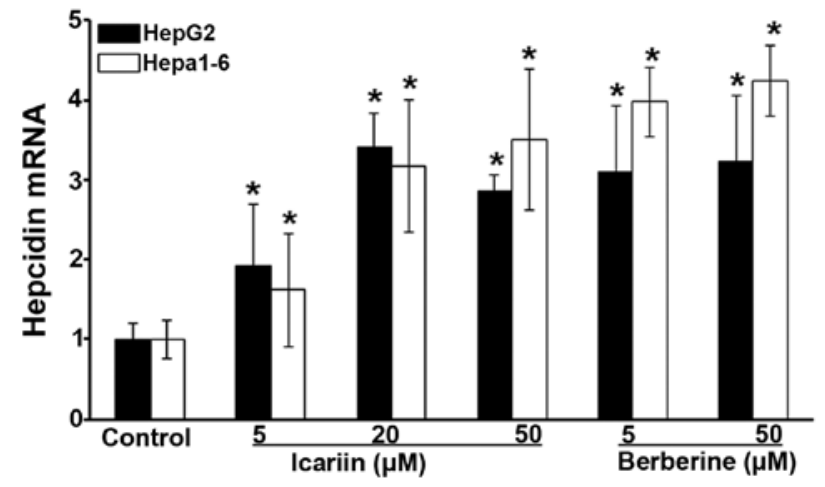

Figure 1. Assessment of hepcidin expression in hepatocytes following treatment with icariin and berberine. Relative hepcidin expression in HepG2 and Hepa 1-6 cells following treatment with the compounds at various concentrations ( $n=4$ replicates). Cells were first seeded in 6-well plates and then treated with the compounds at various concentrations, as indicated. Following treatment for $24 \mathrm{~h}$, RNA was extracted for following qPCR assay. The relative hepcidin level in the untreated cells was defined as 1 for both the HepG2 and Hepa 1-6 cells. GAPDH was used as an internal control. " $\mathrm{P}<0.05$, compared with untreated control.

were further demonstrated by the results of RT-qPCR, and we therefore selected these two compounds for further detailed evaluation.

Icariin and berberine regulate hepcidin expression through Stat 3 and Smad1/5/8 signaling. To elucidate the molecular mechanisms responsible for the promotion of hepcidin expression by icariin and berberine, we explored the signaling pathways involved in the regulation of hepcidin expression. Although the regulation of hepcidin expression is rather complex, two signaling pathways are known to predominantly control hepcidin expression under normal and pathological conditions: the Stat3 pathway and the Smad1/5/8 pathway $(6,8)$. Upon activation of one or both of these pathways, hepcidin expression is expected to rise, which inhibits dietary iron absorption, as well as iron egress from macrophages $(6,8)$. Thus, in this study, we examined the activation of the Stat3 pathway and the Smad1/5/8 pathway in HepG2 cells following treatment with icariin or berberine. As shown in Fig. 2A, both icariin and berberine were found to markedly increase the p-Stat 3 and p-Smad1/5/8 levels compared to the untreated control cells. Since IL-6 has been shown to increase hepcidin expression by activating the Stat 3 signaling pathway $(32,33)$, it was used in this study as a positive control to increase the p-Stat3 level (Fig. 2A). In addition, a compound (namely wogonin) that did not alter hepcidin expression was used as a negative control (Fig. 2A).

Furthermore, the dose-dependent effects of icariin and berberine on Stat 3 and Smad1/5/8 were examined. As illustrated in Fig. 2B, icariin was observed to increase the level of p-Stat 3 , particularly at $50 \mu \mathrm{M}$. Icariin was also found to increase the level of $\mathrm{p}-\mathrm{Smad} 1 / 5 / 8$, relative to the untreated cells, particularly at $50 \mu \mathrm{M}$ (Fig. 2B). The quantitative analysis of Stat 3 phosphorylation and Smad1/5/8 phosphorylation is shown in Fig. $2 \mathrm{C}$ and $\mathrm{D}$, respectively. These observations suggest that icariin upregulates hepcidin expression through both the Stat 3 and Smad1/5/8 pathways. Moreover, icariin at a higher concentration (at $50 \mu \mathrm{M}$ ) exhibited a greater ability to activate 
A
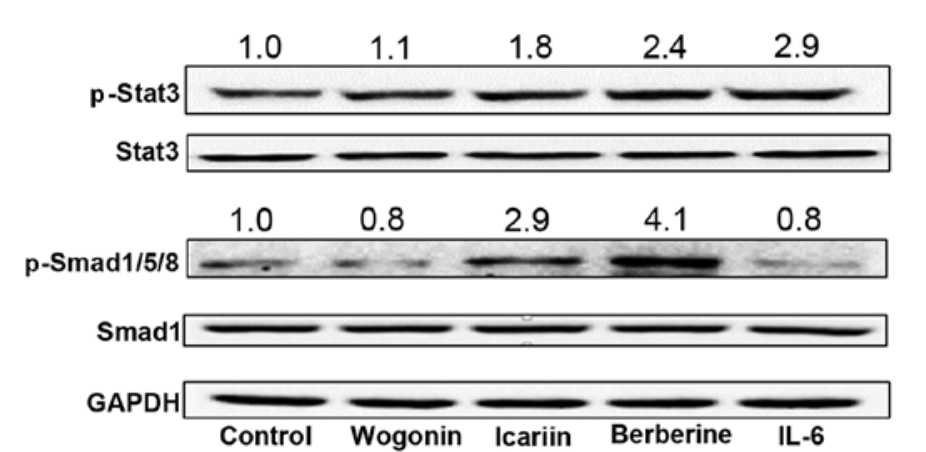

B

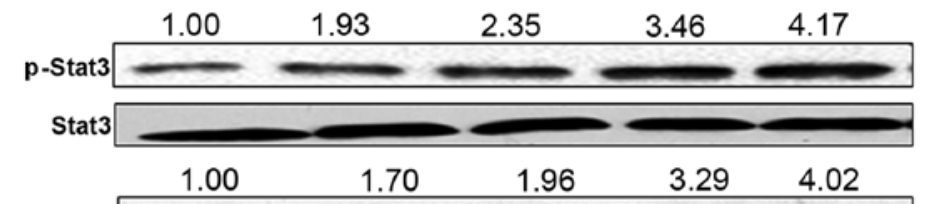

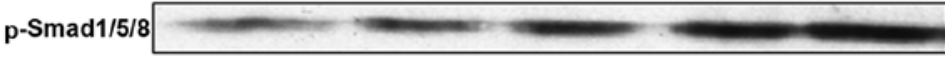

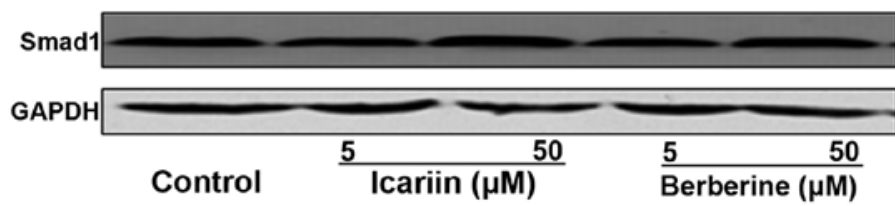

C
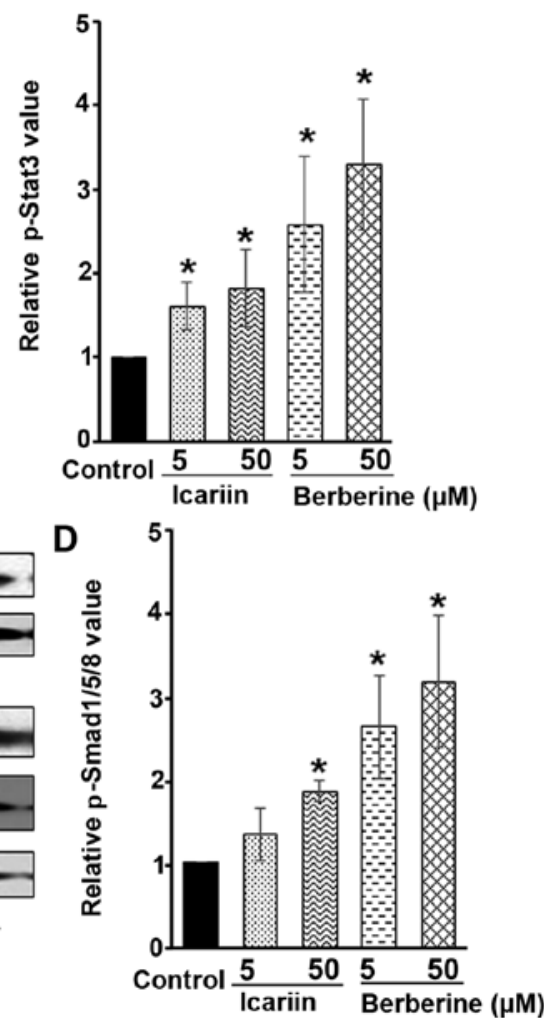

Figure 2. Alterations in phosphorylated (p-)Stat3 and p-Smad1/5/8 levels in HepG2 cells following treatment with icariin and berberine. (A) Cells were treated with icariin, berberine or wogonin at $5 \mu \mathrm{M}$ or with interleukin (IL)- 6 at $100 \mathrm{ng} / \mathrm{ml}$ for $24 \mathrm{~h}$. Cells were then collected for total protein extraction. The changes in $\mathrm{p}$-Stat 3 and $\mathrm{p}$-Smad1/5/8 concentrations were determined by western blot analysis. (B) HepG 2 cells were treated with icariin and berberine at 5 or $50 \mu \mathrm{M}$ for $24 \mathrm{~h}$, followed by western blot analysis. Quantification of (C) Stat3 phosphorylation and (D) Smad1/5/8 phosphorylation levels are shown following normalization to total Stat 3 and total Smad1 ( $\mathrm{n}=3$ replicates). GAPDH was used as an internal control. * $\mathrm{P}<0.05$, compared with untreated control.

the Stat 3 and Smad1/5/8 pathways than at the lower concentration (at $5 \mu \mathrm{M}$ ), in parallel to the greater induction of hepcidin expression observed with $50 \mu \mathrm{M}$ icariin (Fig. 1). Similar to icariin, berberine was also demonstrated to activate both the Stat 3 and Smad1/5/8 pathways, as indicated by a significant increase in the levels of p-Stat 3 and p-Smad1/5/8 (Fig. 2A-D). It should be noted that neither icariin nor berberine altered the basal levels of total Stat3 and Smad1, as shown by the results of western blot analysis (Fig. 2A and B). Taken together, these results demonstrated that the promotion of hepcidin expression by icariin and berberine was due to the activation of both the Stat 3 and Smad1/5/8 pathways; namely, these two signaling pathways jointly promoted hepcidin expression in hepatocytes in response to treatment with icariin and berberine. It should also be noted that it is not possible to exclude the involvement of other pathways which may also have been affected by icariin and berberine.

Icariin regulates hepatic hepcidin expression in mice. To determine whether the in vitro effects of icariin and berberine can be reproduced in vivo, we administered both compounds to mice. As shown in Fig. 3A, consistent with the in vitro results, icariin enhanced hepatic hepcidin mRNA expression by approximately $50 \%(\mathrm{P}<0.05) 6 \mathrm{~h}$ following the administration of icariin, and a maximum induction $(>2.5$-fold, $\mathrm{P}<0.05)$ was observed on day 2 post-icariin administration, compared with the untreated mice (Fig. 3A). The stimulatory effects on hepatic hepcidin expression declined at later time points (Fig. 3A). As a result of alterations in hepatic hepcidin expression, serum iron levels were correspondingly altered, as shown in Fig. 3B. The serum iron concentration was markedly reduced at $6 \mathrm{~h}$ by approximately $40 \%$ in the mice treated with icariin, compared with the untreated mice (Fig. 3B; $\mathrm{P}<0.05$ ). The serum iron concentration was consecutively lower following treatment with icariin compared to the controls on days 2, 4 and $8(\mathrm{P}<0.05)$, reaching similar levels to those of the untreated mice on day 16 (Fig. 3B). Subject to the changes in hepatic hepcidin levels, the spleen iron concentrations were also altered accordingly. As shown in Fig. 3C, the total spleen iron levels were continuously higher following treatment with icariin than those of the untreated mice from $6 \mathrm{~h}$ to the end of the administration on day $16(\mathrm{P}<0.05)$. By contrast, the changes in total hepatic iron levels were less significantly altered upon icariin administration than the spleen iron levels, although they were also higher compared to the controls (Fig. 3D; P>0.05); these results are in agreement with those of previous studies showing the constant hepatic iron content in response to external stimuli $(28,29)$. Notably, berberine did not promote hepcidin expression in mice (Fig. 4A), and no significant alterations in serum, liver and spleen iron levels were observed (Fig. 4B-D); the in vivo results obtained for hepcidin expression were inconsistent with the in vitro results described above.

To further investigate our in vivo findings, ligustrazine (which showed a limited ability to alter hepcidin expres- 

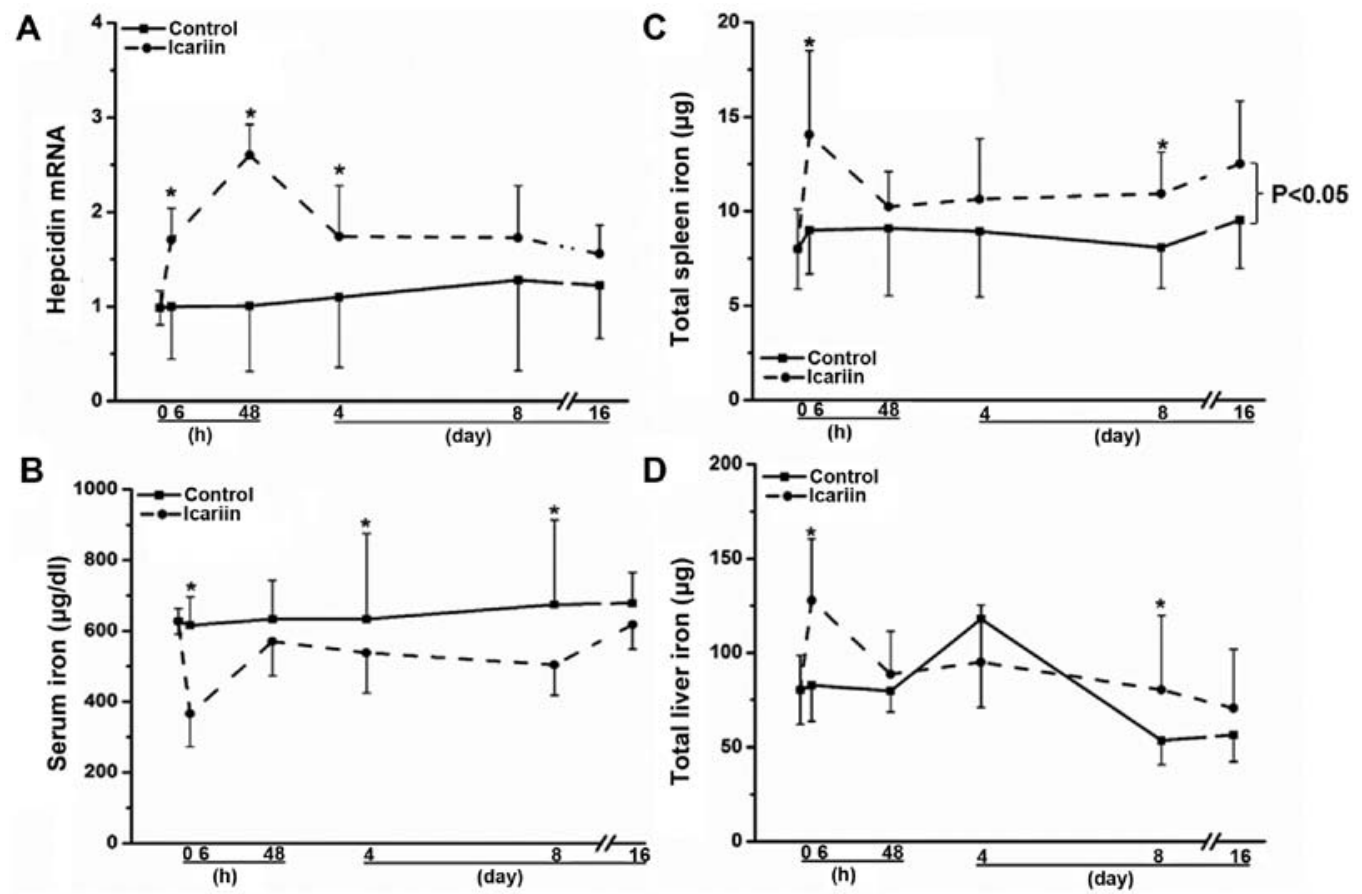

Figure 3. Hepatic hepcidin mRNA expression and changes in iron status in mice treated with icariin. Mice were treated with icariin at $100 \mathrm{mg} / \mathrm{kg}$ body weight, and were sacrificed at different time points, as indicated. Blood and organ specimens were collected for analyses. (A) Relative hepatic hepcidin expression was revealed by RT-qPCR ( $n=8$ mice per group). The relative hepcidin level in the untreated mice (the control group) was defined as 1 . GAPDH was used as an internal control for RT-qPCR. Levels of (B) serum iron, (C) spleen iron and (D) liver iron at each time point following icariin treatment were assayed (n=8 mice per group). ${ }^{*} \mathrm{P}<0.05$, compared with the corresponding untreated mice at the same time points. A bracket with a P-value is presented on the right side showing the overall trend.
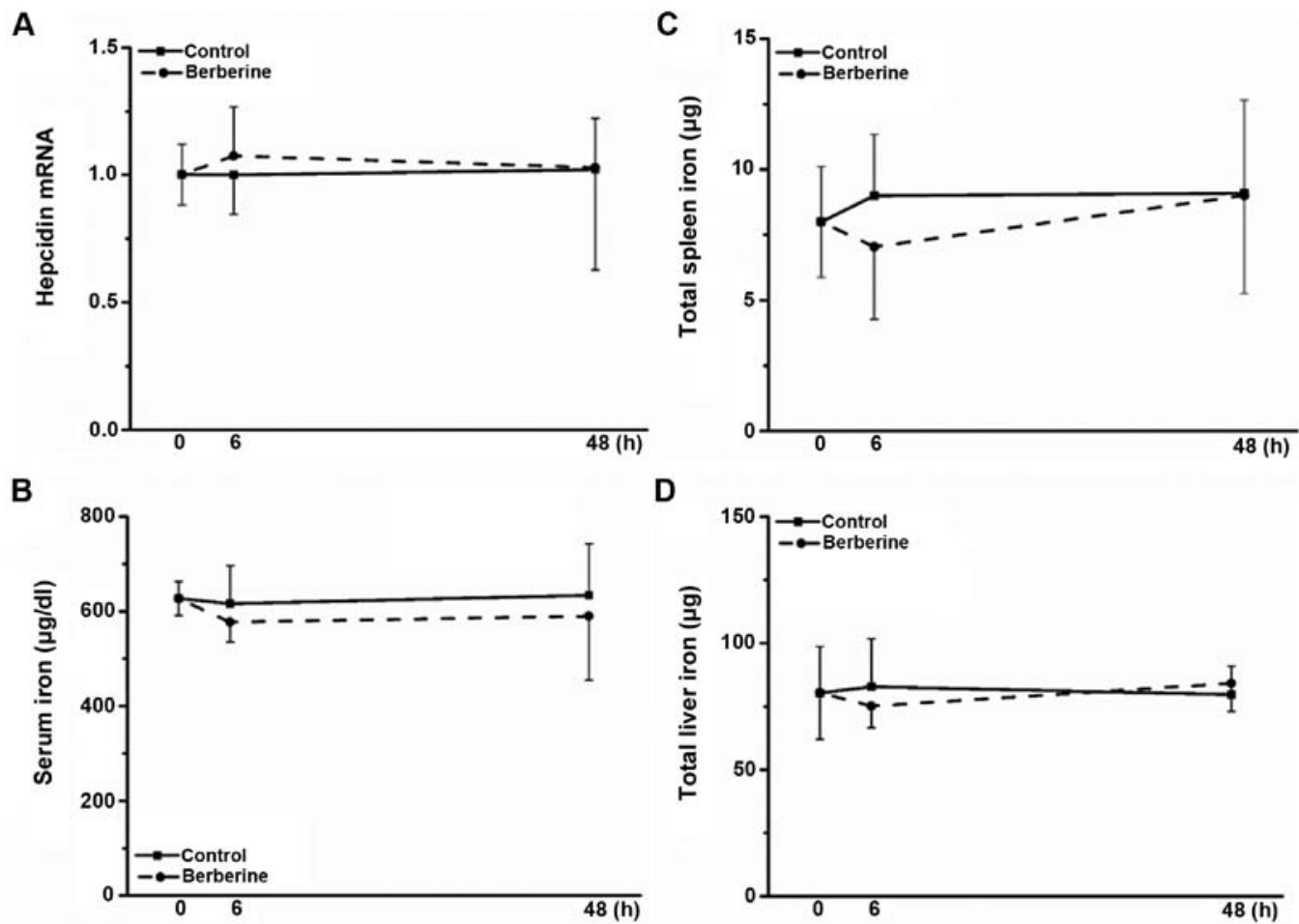

Figure 4. Changes in hepcidin and iron levels in mice treated with berberine. Mice were treated with berberine at $100 \mathrm{mg} / \mathrm{kg}$ body weight. Mice were then sacrificed at different time points for the following assays. (A) Hepatic hepcidin expression levels were determined by RT-qPCR ( $\mathrm{n}=8 \mathrm{mice}$ per group). GAPDH was used as an internal control. Levels of (B) serum iron, (C) spleen iron and (D) liver iron were also determined ( $\mathrm{n}=8$ mice per group).

sion in vitro, as shown in Table I) was administered to the mice as a control. Ligustrazine did not alter the hepcidin expression levels or the body iron status (data not shown). Furthermore,
LPS was used as a positive control to induce hepcidin expression in the mice, as previously described (29). It has been previously established that LPS increases hepcidin expression 


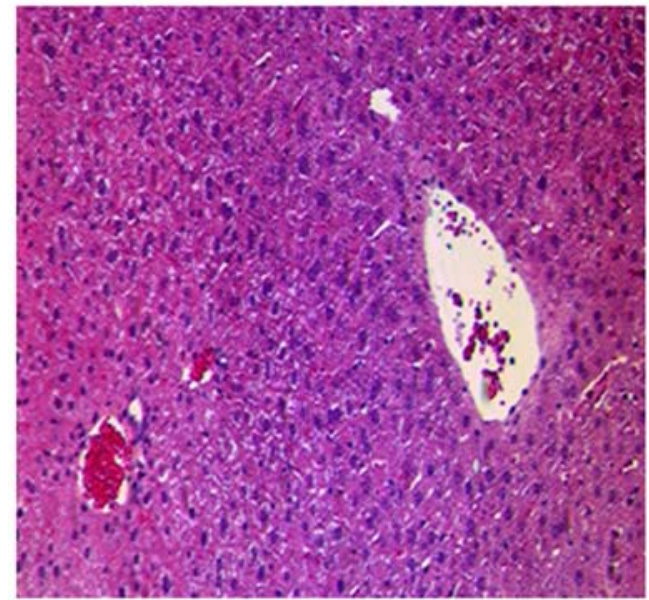

Control

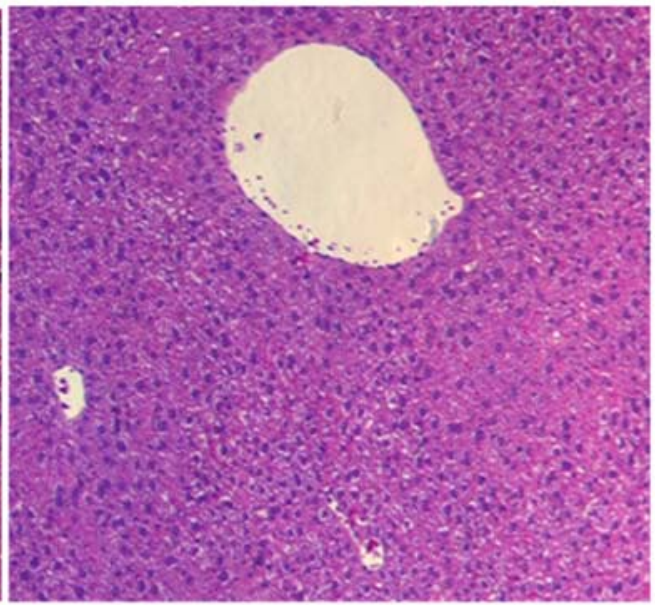

Icariin

Figure 5. Histological examination of liver specimens. Mice were treated with icariin at $100 \mathrm{mg} / \mathrm{kg}$ body weight for $48 \mathrm{~h}$. Liver specimens were subsequently fixed for histological examination. The representative images of hematoxylin and eosin (H\&E) stained liver sections are presented. Original magnification, x100.

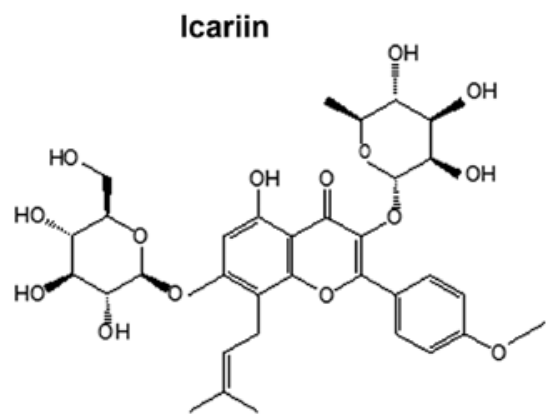

Epimedin B

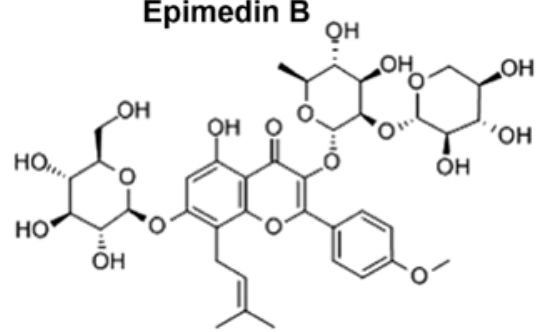

Epimedin A<smiles>COc1ccc(-c2oc3c(CC=C(C)C)c(O[C@@H]4O[C@H](CO)[C@@H](O)[C@H](O)[C@H]4O)cc(O)c3c(=O)c2O[C@@H]2O[C@H](C)[C@@H](O)[C@H](O)[C@H]2O[C@H]2O[C@H](C)[C@@H](O)[C@H](O)[C@H]2O)cc1</smiles>

Epimedin C<smiles>COc1ccc(-c2oc3c(CC=C(C)C)c(OC4OC(CO)[C@@H](O)[C@H](O)C4O)cc(O)c3c(=O)c2O[C@H]2OC(C)[C@@H](O)[C@H](O)[C@H]2OC2OC(C)[C@@H](O)[C@H](O)C2O)cc1</smiles>

Figure 6. Molecular structure of icariin and its analogues, epimedin A, B and C.

through an inflammatory mechanism (34-36). Exposure to LPS significantly increased hepatic hepcidin expression and there were corresponding alterations in body iron status (data not shown). These data further supported our findings regarding the icariin-mediated regulation of hepcidin expression.

No significant hepatotoxicity was observed in the liver specimens from the mice administered icariin. As shown in Fig. 5, no noticeable alterations (no disordered hepatic cords or enlarged central veins) were observed in the liver specimens obtained from the mice treated with icariin for $48 \mathrm{~h}$. This piece of evidence based on histological examination indicated the safety of icariin for in vivo administration.

To further examine the regulatory effects of icariin on hepcidin expression, 3 analogues of icariin, epimedin A, B and $\mathrm{C}$ (which harbor structural similarities to icariin, as depicted in Fig. 6) were also administered to the mice. As shown in Fig. 7A, epimedin A, B and C all significantly increased hepatic hepcidin expression, particularly epimedin $\mathrm{B}$ and $\mathrm{C}(\mathrm{P}<0.05)$. As a result, the serum, spleen and hepatic iron levels were altered accordingly, with more potent effects being observed following the administration of epimedin C (Fig. 7B-D). Collectively, these results suggested that icariin and its analogues exhibited a robust ability to modulate hepatic hepcidin concentrations in mice, which were associated with corresponding alterations in systemic iron levels.

Icariin fails to alter body iron content in Hampl ${ }^{-/}$mice. To confirm the above-mentioned finding that icariin regulates iron homeostasis by altering hepatic hepcidin expression, we used $\mathrm{Hamp1}^{-/-}$mice, as previously described (26). Hamp1 $1^{-/-}$mice do not produce functional hepcidin, and these mice thus develop severe iron overload in the serum and organs after weaning. To more clearly demonstrate changes in serum iron levels in the animals that were already iron-loaded, we depleted body iron 

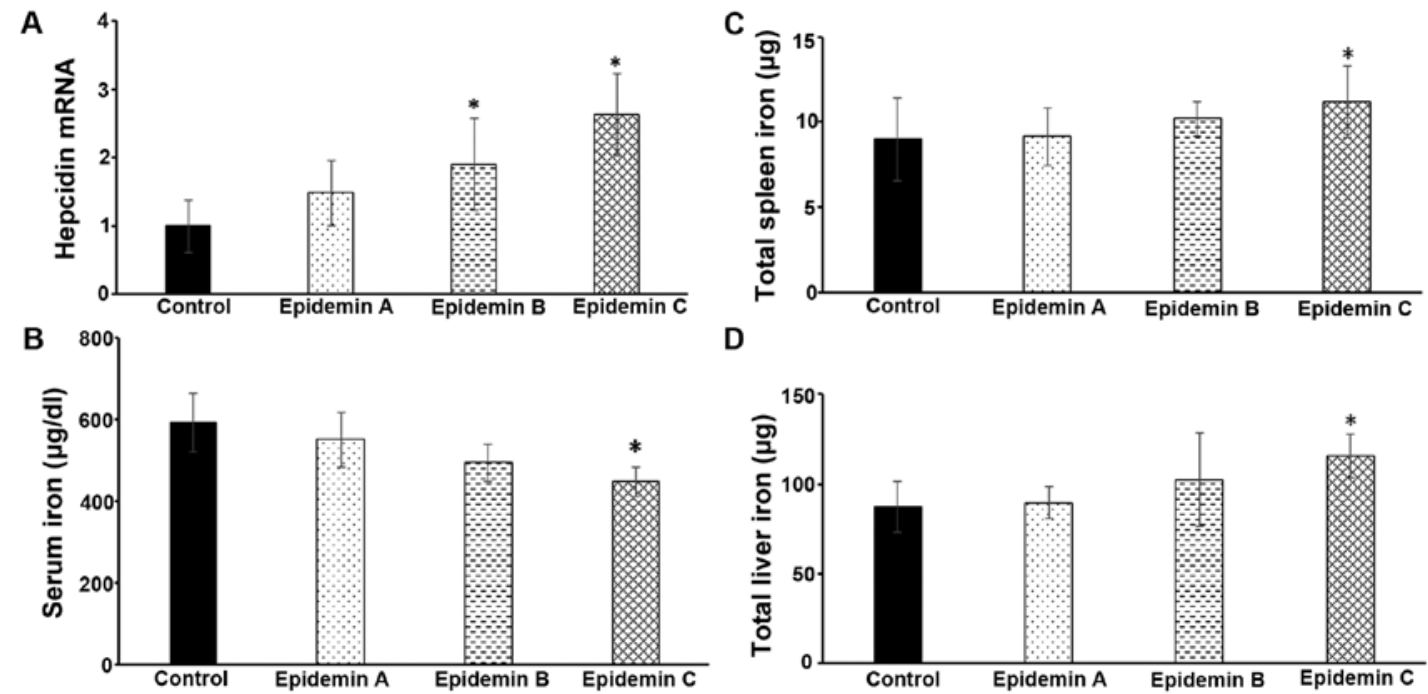

Figure 7. Changes in hepcidin expression and body iron content in mice following the administration of epimedin A, B and C. Mice were treated with epimedin A, B and C at $100 \mathrm{mg} / \mathrm{kg}$ body weight for $48 \mathrm{~h}$. Thereafter, (A) hepatic hepcidin expression, (B) serum iron, (C) total spleen iron and (D) total liver iron levels were examined $(\mathrm{n}=8)$. "P<0.05, compared with untreated control.
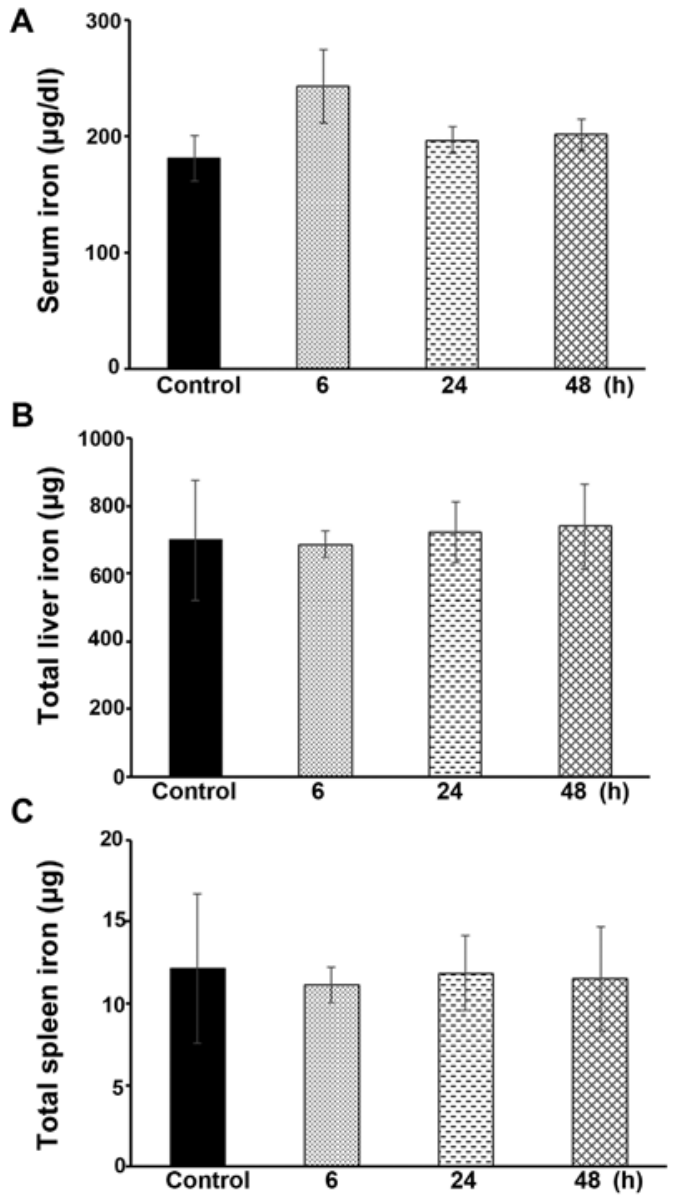

Figure 8. Changes in iron content $\mathrm{Hamp1}^{-/-}$mice upon icariin treatment. Hamp1 ${ }^{-1-}$ mice were treated with icariin at $100 \mathrm{mg} / \mathrm{kg}$ body weight for 6,24 and $48 \mathrm{~h}$. Iron levels in (A) serum, (B) liver and (C) spleen were determined ( $\mathrm{n}=8$ mice per group).

by placing these mice on a low-iron diet. In other words, this regime would in fact increase the sensitivity of these Hamp1 ${ }^{-1-}$ mice to the administration of the compounds. Following iron depletion, we observed a significant reduction in the serum iron levels in the Hamp1 ${ }^{-/-}$mice (data not shown). As shown in Fig. 8A, the serum iron levels were not significantly altered in the Hamp1 ${ }^{-/-}$mice following the administration of icariin at the same dose as that administered to the Wt ICR mice, as described above, i.e., at $100 \mathrm{mg} / \mathrm{kg}$ body weight, for 6,24 or $48 \mathrm{~h}$ ( $\mathrm{P}>0.05)$. Moreover, there were no significant changes observed in the total hepatic or spleen iron levels in the icariintreated Hamp1 $1^{-/}$mice, compared with the untreated control mice (Fig. 8B and C). These data further demonstrate that icariin predominantly targets hepatic hepcidin expression in order to modulate iron homeostasis.

\section{Discussion}

The primary therapies for iron overload-related diseases are bloodletting (phlebotomy) and iron chelation. However, these therapies have significant limitations. For instance, repeated phlebotomy may lead to anemia (37) and long-term administration of iron chelators may also cause infections, gastrointestinal disorders and skin damage (38). Therefore, increasing hepcidin expression is a potential alternative strategy to relieve iron overload in $\mathrm{HH}$ and $\beta$-thalassemia, and also to diminish the severity of $\beta$-thalassemia by reducing ineffective erythropoiesis. Hepcidin analogue peptides (minihepcidins) have been investigated for their ability to relieve iron overload in hepcidin-KO mice, although they are not orally available (27). It has been demonstrated that genistein, a small molecule, increases hepcidin expression through Stat3-dependent and Smad4-dependent pathways in HepG2 cells (39). A recent chemical screen also identified 16 small molecules that induce hepcidin expression in human HepG2 cells, although animal studies were not performed (40). In comparison with proteins and synthesized chemicals, natural compounds from medicinal plants have novel features, including replete supply, high stability and low toxicity $(41,42)$. A previous study suggested that some medicinal plant extracts may repress hepcidin expression (43). Among the 16 medicinal plant extracts tested in that 
study (43), Caulis Spatholobi (CS; also known as Jixueteng, the stem of Spatholobus suberectus Dann) exhibited the most potent inhibitory effect on hepcidin expression. However, to the best of our knowledge, no natural compounds or extracts from traditional Chinese medicinal plants have been demonstrated to increase hepcidin levels thus far. In the present study, we screened 12 pure natural compounds extracted from traditional Chinese medicinal plants, namely, propyl gallate, resveratrol, astragaloside, curcumin, paeoniflorin, ligustrazine, ferulic acid, ginsenoside Rb1, wogonin, liquiritin, berberine and icariin to examine their effects on hepcidin expression. According to the Chinese Pharmacopoeia (21), these compounds may have the ability to improve blood supply, implying that they may potentially enhance red blood cell formation and alter iron metabolism. In this study, we set up a pipeline to identify compounds that have the potential to modulate hepcidin expression. Firstly, we used a luciferase reporter system to screen these compounds in two cell lines. The results indicated that only wogonin, icariin and berberine promoted hepcidin expression by $>2$-fold in both cell lines. Thereafter, wogonin, icariin and berberine were subjected to RT-qPCR analysis. The results revealed that only icariin and berberine, but not wogonin (data not shown), significantly increased hepcidin expression in both cell lines. In subsequent animal experiments, only icariin, but not berberine, increased hepatic hepcidin expression. The icariin analogues epimedin A, B and C also increased hepcidin expression. Epimedin A has the hydroxyl in the rhamnose $\mathrm{C}-2$ position substituted by glucose, whereas in epimedin $\mathrm{B}$ and $\mathrm{C}$, xylose and rhamnose, respectively, are substituted at this position. Our combined data suggested that the parental flavone framework likely dictates the structure-activity relationship (SAR) and that the glycosylation derivatives may affect the SAR and consequently affect the way in which the compound regulates hepcidin expression.

We further investigated the molecular mechanisms responsible for the stimulatory effects of icariin on hepcidin expression. The Stat 3 and Smad1/5/8 signaling pathways are two important pathways which regulate hepatic hepcidin expression under physiological and pathological conditions $(4,44)$. We found that both the Stat 3 and Smad1/5/8 pathways were activated by icariin. Furthermore, in support of the data obtained from the animal experiments, the iron levels in the Hamp1 $1^{-/}$mice did not respond to icariin, suggesting that icariin affects iron levels through a hepcidin-dependent mechanism. Additionally, icariin at the dose used did not cause noticeable toxicity to the liver. Icariin is a flavonol glycoside purified from plants of the genus Epimedium (45), and previous studies have documented that it has multiple functions which include roles in reducing oxidative stress, limiting apoptosis, protecting the cardiovascular system and promoting angiogenesis (46-49). The present study is the first, to the very best of our knowledge, to reveal that icariin regulates iron metabolism by modulating hepcidin expression.

In conclusion, this study demonstrated that icariin and icariin-like natural compounds purified from traditional Chinese medicinal plants enhanced hepatic hepcidin transcription. At the molecular level, icariin was demonstrated to simultaneously activate Stat 3 signaling and Smad1/5/8 signaling, increasing hepcidin expression. Experiments using mice confirmed the stimulatory effect of icariin on hepatic hepcidin. In addition, according to previous studies on natural Chinese medicinal herbs (50-52), the compounds similar to icariin would be rapidly eliminated with a short half-life if administered orally to animals. By contrast, these compounds revealed great bioavailability with a long half-life when administered intraperitoneally (53). Therefore, these results demonstrate that natural compounds (such as icariin) may be developed into therapies (e.g., injection) which ameliorate iron overload by restricting dietary iron absorption and iron egress from macrophages in iron overload-related diseases with hepcidin deficit. This pilot study represents a promising strategy for the treatment of iron overload-related diseases (such as $\mathrm{HH}$ and $\beta$-thalassemia) through the administration of natural compounds, particularly extracts from traditional Chinese medicinal plants.

\section{Acknowledgements}

The present study was supported by a grant under the National '973' Program (grant no. 2014CB932000), the Strategic Priority Research Program of the Chinese Academy of Sciences (grant no. XDB14000000) and grants from the National Natural Science Foundation of China (grant nos. 21425731, 21377159, 21321004, 21207152 and 21407169). We thank Dr Sophie Vaulont and Dr Tomas Ganz for providing the Hamp $^{-/}$mice. We would like to thank Dr Jun Liu for making suggestions on icariin-like compounds. We would also like to thank all the laboratory members for their great assistance with the experiments and reagents.

\section{References}

1. Hentze MW, Muckenthaler MU and Andrews NC: Balancing acts: molecular control of mammalian iron metabolism. Cell 117: 285-297, 2004

2. Wu Y and Brosh RM Jr: DNA helicase and helicase-nuclease enzymes with a conserved iron-sulfur cluster. Nucleic Acids Res 40: 4247-4260, 2012.

3. Brzóska K, Meczyńska S and Kruszewski M: Iron-sulfur cluster proteins: electron transfer and beyond. Acta Biochim Pol 53: 685-691, 2006

4. Evstatiev R and Gasche C: Iron sensing and signalling. Gut 61: 933-952, 2012.

5. Ganz T and Nemeth E: Hepcidin and disorders of iron metabolism. Annu Rev Med 62: 347-360, 2011.

6. Ganz T and Nemeth E: Hepcidin and iron homeostasis. Biochim Biophys Acta 1823: 1434-1443, 2012.

7. Camaschella C: Understanding iron homeostasis through genetic analysis of hemochromatosis and related disorders. Blood 106: 3710-3717, 2005

8. Ganz T: Systemic iron homeostasis. Physiol Rev 93: 1721-1741, 2013.

9. Tanno T, Bhanu NV, Oneal PA, Goh SH, Staker P, Lee YT, Moroney JW, Reed CH, Luban NL, Wang RH, et al: High levels of GDF15 in thalassemia suppress expression of the iron regulatory protein hepcidin. Nat Med 13: 1096-1101, 2007.

10. Tanno T, Porayette P, Sripichai O, Noh SJ, Byrnes C, Bhupatiraju A, Lee YT, Goodnough JB, Harandi O, Ganz T, et al: Identification of TWSG1 as a second novel erythroid regulator of hepcidin expression in murine and human cells. Blood 114: 181-186, 2009.

11. Kautz L, Jung G, Valore EV, Rivella S, Nemeth E and Ganz T: Identification of erythroferrone as an erythroid regulator of iron metabolism. Nat Genet 46: 678-684, 2014.

12. Kumar A, Ekavali, Chopra K, Mukherjee M, Pottabathini R and Dhull DK: Current knowledge and pharmacological profile of berberine: an update. Eur J Pharmacol 761: 288-297, 2015.

13. Ghosh S, Banerjee S and Sil PC: The beneficial role of curcumin on inflammation, diabetes and neurodegenerative disease: a recent update. Food Chem Toxicol 83: 111-124, 2015. 
14. Mancuso $\mathrm{C}$ and Santangelo R: Ferulic acid: pharmacological and toxicological aspects. Food Chem Toxicol 65: 185-195, 2014.

15. Ren S, Zhang H, Mu Y, Sun M and Liu P: Pharmacological effects of astragaloside IV: a literature review. J Tradit Chin Med 33: 413-416, 2013.

16. Jiang YR and Chen KJ: Pharmacological roles of ligustrazine in cardio-/cerebrovascular systems and its progress in researches of clinical application. Zhongguo Zhong Xi Yi Jie He Za Zhi 33: 707-711, 2013 (In Chinese).

17. Chen Y, Huang JH, Ning Y and Shen ZY: Icariin and its pharmaceutical efficacy: research progress of molecular mechanism. Zhong Xi Yi Jie He Xue Bao 9: 1179-1184, 2011 (In Chinese).

18. Sun LR, Cao X, Hou FQ, Zhu XH and Gao TM: Progressive studies of paeoniflorin. Zhongguo Zhong Yao Za Zhi 33: 2028-2032, 2008 (In Chinese).

19. Jia JM, Wang ZQ, Wu LJ and Wu YL: Advance of pharmacological study on ginsenoside Rb1. Zhongguo Zhong Yao Za Zhi 33: 1371-1377, 2008 (In Chinese).

20. Tai MC, Tsang SY, Chang LY and Xue H: Therapeutic potential of wogonin: a naturally occurring flavonoid. CNS Drug Rev 11: $141-150,2005$

21. Chinese Pharmacopoeia Commission. Pharmacopoeia of the People's Republic of China. China Medical Science Press, Beijing, 2010.

22. Zhang W, Yan ZF, Gao JH, Sun L, Huang XY, Liu Z, Yu SY, Cao CJ, Zuo LJ, Chen ZJ, et al: Role and mechanism of microglial activation in iron-induced selective and progressive dopaminergic neurodegeneration. Mol Neurobiol 49: 1153-1165, 2014.

23. Liu W, Zhang S, Wang L, Qu C, Zhang C, Hong L, Yuan L, Huang Z, Wang Z, Liu S and Jiang G: CdSe quantum dot (QD)-induced morphological and functional impairments to liver in mice. PLoS One 6: e24406, 2011.

24. Chen Y, Wang Z, Xu M, Wang X, Liu R, Liu Q, Zhang Z, Xia T, Zhao J, Jiang G, et al: Nanosilver incurs an adaptive shunt of energy metabolism mode to glycolysis in tumor and nontumor cells. ACS Nano 8: 5813-5825, 2014.

25. Zhang S, Chen Y, Guo W, Yuan L, Zhang D, Xu Y, Nemeth E, Ganz T and Liu S: Disordered hepcidin-ferroportin signaling promotes breast cancer growth. Cell Signal 26: 2539-2550, 2014

26. Lesbordes-Brion JC, Viatte L, Bennoun M, Lou DQ, Ramey G Houbron C, Hamard G, Kahn A and Vaulont S: Targeted disruption of the hepcidin 1 gene results in severe hemochromatosis. Blood 108: 1402-1405, 2006.

27. Ramos E, Ruchala P, Goodnough JB, Kautz L, Preza GC, Nemeth E and Ganz T: Minihepcidins prevent iron overload in a hepcidin-deficient mouse model of severe hemochromatosis. Blood 120: 3829-3836, 2012.

28. Liu S, Suragani RN, Han A, Zhao W, Andrews NC and Chen JJ: Deficiency of heme-regulated eIF2alpha kinase decreases hepcidin expression and splenic iron in $\mathrm{HFE}^{-/-}$mice. Haematologica 93: 753-756, 2008.

29. Liu S, Suragani RN, Wang F, Han A, Zhao W, Andrews NC and Chen JJ: The function of heme-regulated eIF2alpha kinase in murine iron homeostasis and macrophage maturation. J Clin Invest 117: 3296-3305, 2007.

30. Park $\mathrm{CH}$, Valore EV, Waring AJ and Ganz T: Hepcidin, a urinary antimicrobial peptide synthesized in the liver. J Biol Chem 276 7806-7810, 2001.

31. Hou Y, Zhang S, Wang L, Li J, Qu G, He J, Rong H, Ji H and Liu S: Estrogen regulates iron homeostasis through governing hepatic hepcidin expression via an estrogen response element. Gene 511: 398-403, 2012.

32. Nemeth E, Rivera S, Gabayan V, Keller C, Taudorf S, Pedersen BK and Ganz T: IL- 6 mediates hypoferremia of inflammation by inducing the synthesis of the iron regulatory hormone hepcidin. J Clin Invest 113: 1271-1276, 2004.

33. Wrighting DM and Andrews NC: Interleukin-6 induces hepcidin expression through STAT3. Blood 108: 3204-3209, 2006

34. Pigeon C, Ilyin G, Courselaud B, Leroyer P, Turlin B, Brissot P and Loréal O: A new mouse liver-specific gene, encoding a protein homologous to human antimicrobial peptide hepcidin, is overexpressed during iron overload. J Biol Chem 276: 7811-7819, 2001.
35. Poli M,Asperti M,Naggi A,Campostrini N, Girelli D, Corbella M, Benzi M, Besson-Fournier C, Coppin H, Maccarinelli F, et al: Glycol-split nonanticoagulant heparins are inhibitors of hepcidin expression in vitro and in vivo. Blood 123: 1564-1573, 2014

36. Lee P, Peng H, Gelbart T and Beutler E: The IL-6- and lipopolysaccharide-induced transcription of hepcidin in $H F E$-, transferrin receptor 2-, and beta 2-microglobulin-deficient hepatocytes. Proc Natl Acad Sci USA 101: 9263-9265, 2004.

37. Adams PC and Barton JC: How I treat hemochromatosis. Blood 116: 317-325, 2010.

38. Pennell DJ, Porter JB, Cappellini MD, El-Beshlawy A, Chan LL, Aydinok Y, Elalfy MS, Sutcharitchan P, Li CK, Ibrahim H, et al: Efficacy of deferasirox in reducing and preventing cardiac iron overload in beta-thalassemia. Blood 115: 2364-2371, 2010.

39. Zhen AW, Nguyen NH, Gibert Y, Motola S, Buckett P, Wessling-Resnick M, Fraenkel E and Fraenkel PG: The small molecule, genistein, increases hepcidin expression in human hepatocytes. Hepatology 58: 1315-1325, 2013.

40. Gaun V, Patchen B, Volovetz J, Zhen AW, Andreev A, Pollastri MP and Fraenkel PG: A chemical screen identifies small molecules that regulate hepcidin expression. Blood Cells Mol Dis 53: 231-240, 2014.

41. Kennedy DO and Wightman EL: Herbal extracts and phytochemicals: plant secondary metabolites and the enhancement of human brain function. Adv Nutr 2: 32-50, 2011

42. Ziegler G, Ploch M, Miettinen-Baumann A and Collet W: Efficacy and tolerability of valerian extract LI 156 compared with oxazepam in the treatment of non-organic insomnia - a randomized, double-blind, comparative clinical study. Eur J Med Res 7: 480-486, 2002.

43. Guan Y, An P, Zhang Z, Zhang F, Yu Y, Wu Q, Shi Y, Guo X, Tao Y and Wang F: Screening identifies the Chinese medicinal plant Caulis Spatholobi as an effective HAMP expression inhibitor. J Nutr 143: 1061-1066, 2013.

44. Bartnikas TB and Fleming MD: A tincture of hepcidin cures all: the potential for hepcidin therapeutics. J Clin Invest 120 : 4187-4190, 2010

45. Ma H, He X, Yang Y, Li M, Hao D and Jia Z: The genus Epimedium: an ethnopharmacological and phytochemical review. J Ethnopharmacol 134: 519-541, 2011.

46. Xu CQ, Liu BJ, Wu JF, Xu YC, Duan XH, Cao YX and Dong JC: Icariin attenuates LPS-induced acute inflammatory responses: involvement of PI3K/Akt and NF-kappaB signaling pathway. Eur J Pharmacol 642: 146-153, 2010.

47. Li WW, Gao XM, Wang XM, Guo $\mathrm{H}$ and Zhang BL: Icariin inhibits hydrogen peroxide-induced toxicity through inhibition of phosphorylation of JNK/p38 MAPK and p53 activity. Mutat Res 708: 1-10, 2011.

48. Song YH, Cai H, Gu N, Qian CF, Cao SP and Zhao ZM: Icariin attenuates cardiac remodelling through down-regulating myocardial apoptosis and matrix metalloproteinase activity in rats with congestive heart failure. J Pharm Pharmacol 63: 541-549, 2011.

49. ChungBH,KimJD,Kim CK,Kim JH,WonMH,LeeHS,Dong MS, Ha KS, Kwon YG and Kim YM: Icariin stimulates angiogenesis by activating the MEK/ERK- and PI3K/Akt/eNOS-dependent signal pathways in human endothelial cells. Biochem Biophys Res Commun 376: 404-408, 2008.

50. Chen Y, Wang J, Jia X, Tan X and Hu M: Role of intestinal hydrolase in the absorption of prenylated flavonoids present in Yinyanghuo. Molecules 16: 1336-1348, 2011.

51. Chen Y,Zhao YH, Jia XB and Hu M: Intestinal absorption mechanisms of prenylated flavonoids present in the heat-processed Epimedium koreanum Nakai (Yin Yanghuo). Pharm Res 25: 2190-2199, 2008.

52. Liu M, Liu H, Lu X, Li C, Xiong Z and Li F: Simultaneous determination of icariin, icariside II and osthole in rat plasma after oral administration of the extract of Gushudan (a Chinese compound formulation) by LC-MS/MS. J Chromatogr B Analyt Technol Biomed Life Sci 860: 113-120, 2007.

53. Xu W, Sui ZG and Zhong W: Pharmacokinetics of icariin and its two metabolites in rats. The Third China Pharmacist Assembly pp1-5, 2011. 